\title{
Extensions of the charged Riemannian Penrose inequality
}

\author{
Marcus Khuri ${ }^{1}$, Gilbert Weinstein ${ }^{2}$ and Sumio Yamada ${ }^{3}$ \\ ${ }^{1}$ Department of Mathematics, Stony Brook University, Stony Brook NY 11794, USA \\ ${ }^{2}$ Physics Department and Department of Computer Science and Mathematics, Ariel \\ University of Samaria, Ariel 40700, Israel \\ ${ }^{3}$ Department of Mathematics, Gakushuin University, Tokyo171-8588, Japan \\ E-mail: khuri@math.sunysb.edu, gilbertw@ariel.ac.il and yamada@math.gakushuin. \\ ac.jp
}

Received 23 October 2014, revised 12 November 2014

Accepted for publication 17 November 2014

Published 14 January 2015

\begin{abstract}
In this paper we investigate the extension of the charged Riemannian Penrose inequality to the case where charges are present outside the horizon. We prove a positive result when the charge densities are compactly supported, and present a counterexample when the charges extend to infinity. We also discuss additional extensions to other matter models.
\end{abstract}

Keywords: Penrose inequality, charge density, black holes

(Some figures may appear in colour only in the online journal)

\section{Introduction}

In [15], we proved the Riemmanian Penrose inequality with charge for multiple black holes.

Theorem 1.1. Let $(M, g, E, B)$ be a strongly asymptotically flat initial data set for the Einstein-Maxwell equations with outermost minimal surface boundary of area $A=4 \pi \rho^{2}$, with ADM mass $m$, and total charge q, satisfying the charged dominant energy condition and the Maxwell constraints without charged matter. Then

$$
\rho \leqslant m+\sqrt{m^{2}-q^{2}}
$$

with equality if and only if the data set arises as the canonical slice of the ReissnerNordström spacetime.

Here $M$ is a three-dimensional manifold, $g$ a Riemannian metric on $M, E$ and $B$ vector fields on $M$, and $\rho$ is called the area radius of the outermost minimal surface. When $|q| \geqslant \rho$, 
the positive mass theorem with charge [8], $m \geqslant|q|$, immediately implies (1). Thus to prove theorem 1.1, it was sufficient to prove (1) when $|q| \leqslant \rho$, which was accomplished with the following result.

Theorem 1.2. Let $(M, g, E, B)$ be a strongly asymptotically flat initial data set for the Einstein-Maxwell equations with outermost minimal surface boundary of area $A=4 \pi \rho^{2}$, with ADM mass $m$, and total charge q, satisfying the charged dominant energy condition and the Maxwell constraints without charged matter. If $|q| \leqslant \rho$, then

$$
m \geqslant \frac{1}{2}\left(\rho+\frac{q^{2}}{\rho}\right),
$$

with equality if and only if the data set arises as the canonical slice of the ReissnerNordström spacetime.

We proved this theorem using a conformal flow method adapted from [1]. In [24], a counterexample to (2) based on the Majumdar-Papapetrou solutions was constructed when $\rho<|q|$, showing that the hypothesis $|q| \leqslant \rho$ is necessary in theorem 1.2. In this paper we examine whether the hypothesis $\operatorname{div} E=\operatorname{div} B=0$, i.e. the absence of charges outside the horizon, is necessary. We will prove the following two theorems; a positive and a negative result.

Theorem 1.3. Let $(M, g, E, B)$ be a strongly asymptotically flat initial data set for the Einstein-Maxwell equations with outermost minimal surface boundary of area $A=4 \pi \rho^{2}$, with ADM mass $m$, and total charge $q$, satisfying the charged dominant energy condition. If $\operatorname{div} E$ and $\operatorname{div} B$ are compactly supported, then (1) holds.

Theorem 1.4. There is a spherically symmetric counterexample to (1) with $B=0$, satisfying all the conditions of theorem 1.3 except that $\operatorname{div} E$ is not compactly supported.

The existence of a spherically symmetric counterexample was conjectured in [17]. Note that in theorem 1.3 the rigidity statement has been omitted. In fact, we show that a counterexample to rigidity exists.

Proposition 1.5. There is a non-trivial arbitrarily small spherically symmetric perturbation of the Reissner-Nordström canonical slice $(M, g, E, 0)$, which coincides with ReissnerNordström outside of an annulus, satisfies all the hypothesis of theorem 1.3 in addition to $|q| \leqslant \rho$, and saturates inequality (2):

$$
m=\frac{1}{2}\left(\rho+\frac{q^{2}}{\rho}\right) .
$$

We also examine the question of whether the conformal flow technique developed in $[15,16]$ can be extended to other matter models. It is expected that for a Riemannian Penrose inequality to hold, the matter model should admit a unique stable static black hole solution. At the moment, we are only aware of two such models, the Einstein-Abelian-Yang-Mills (EAYM), and the Einstein-Maxwell-Dilaton (EMD). We prove a Riemannian Penrose 
inequality for EAYM black holes, and conjecture that a Riemannian Penrose inequality for EMD black holes also holds.

Theorem 1.6. Let $\left(M, g, E_{1}, B_{1}, \ldots, E_{\ell}, B_{\ell}\right)$ be a strongly asymptotically flat initial data set for the EAYM equations with an outermost minimal surface boundary of area $A=4 \pi \rho^{2}$, with ADM mass $m$, and total charge $q$, satisfying the EAYM dominant energy condition (9). If $\operatorname{div} E_{i}$ and $\operatorname{div} B_{i}$ are compactly supported, then (1) holds. If there are no charges outside the horizon then equality holds if and only if the data set arises as the canonical slice of an EAYM Reissner-Nordström spacetime.

Conjecture 1.7. Let $(M, g, E, B, \phi)$ be a strongly asymptotically flat initial data set for the EMD equations with an outermost minimal surface boundary of area $A=4 \pi \rho^{2}$, ADM mass $m$, and total charge $q$, satisfying the EMD dominant energy condition (10). If the charge densities $\operatorname{div}\left(\mathrm{e}^{-2 \phi} E\right)$ and $\operatorname{div} B$ are compactly supported, then

$$
m \geqslant \frac{1}{2} \sqrt{\rho^{2}+2 q^{2}} .
$$

If $\mathrm{e}^{-2 \phi} E$ and $B$ are divergence free, then equality holds if and only if the data set arises as the canonical slice of the Gibbons EMD black hole.

The plan of the paper is as follows. In the next section, we briefly present some of the background, heuristics, and prior work on the Riemannian Penrose inequality without and with charge. In that section we also discuss the dominant energy conditions for EAYM data sets and for EMD data sets, as well as the EMD black hole. In section 3, we prove theorems 1.3, 1.4 and proposition 1.5. In section 4 , we prove theorem 1.6 and discuss conjecture 1.7.

\section{Background}

\subsection{The Penrose inequality}

The Penrose inequality was originally proposed by Penrose as a test of the cosmic censorship conjecture, or more generally a test of the standard picture of gravitational collapse [21, 22]. Consider a strongly asymptotically flat Cauchy surface in a spacetime satisfying the dominant energy condition, with ADM mass $m_{0}$, and containing an event horizon of area $A_{0}=4 \pi \rho_{0}^{2}$, which undergoes gravitational collapse and settles to a Kerr-Newman black hole. Since the ADM mass $m$ of the final state is no greater than $m_{0}$, and since the end state area radius $\rho$ is no less than $\rho_{0}$ by Hawking's area theorem [10], and since for the final state $m \geqslant \rho / 2$ in order to avoid naked singularities, it must have been the case that $m_{0} \geqslant \rho_{0} / 2$ also at the beginning of the evolution. The event horizon is indiscernible in the original slice without knowing the full evolution. However, one may replace the event horizon by the outermost minimal area enclosure of the apparent horizon, the boundary of the region admitting trapped surfaces, and reach the same conclusion. A counterexample to the Penrose inequality would therefore have suggested Cauchy data which leads under the Einstein evolution to naked singularities, while a proof of the inequality could be viewed as evidence in support of cosmic censorship.

\subsection{Penrose's heuristic argument for multiple black holes}

It is usually assumed that the end state of gravitational collapse is a single Kerr-Newman black hole. However, a more appropriate assumption for the end state is a finite number of 
mutually distant Kerr-Newman black holes moving apart with asymptotically constant velocity. This should be the result, if for instance, two distant black holes were initially moving away from each other sufficiently fast. We will now describe the heuristic Penrose argument for the charged Penrose inequality (1) in this setting. It appears that this has not been previously considered in the literature.

Let $m_{i}, A_{i}=4 \pi \rho_{i}^{2}, q_{i}, J_{i}$ denote the ADM masses, horizon areas, total charges, and angular momenta of the end state black holes. Then the total (ADM) mass, horizon area radius, and charge of the end state is $m=\sum m_{i}, \rho=\left(\sum \rho_{i}^{2}\right)^{1 / 2}, q=\sum q_{i}$. The area radius of the Kerr-Newman black hole [4] is given by

$\frac{\rho_{i}^{2}}{2}=m_{i}^{2}-\frac{q_{i}^{2}}{2}+\sqrt{\left(m_{i}^{2}-\frac{q_{i}^{2}}{2}\right)^{2}-\frac{q_{i}^{4}}{4}-J_{i}^{2}} \leqslant m_{i}^{2}-\frac{q_{i}^{2}}{2}+\sqrt{\left(m_{i}^{2}-\frac{q_{i}^{2}}{2}\right)^{2}-\frac{q_{i}^{4}}{4}}$.

It follows that

$$
\rho_{i} \leqslant m_{i}+\sqrt{m_{i}^{2}-q_{i}^{2}}
$$

Let $m_{0}, \rho_{0}, q_{0}$ denote the ADM mass, horizon area radius, and total charge of an initial state. Under the assumption that no charged matter is present, the total charge is conserved $q_{0}=q=\sum q_{i}$. Moreover, by the Hawking area theorem $\rho_{0} \leqslant \rho \leqslant \sum \rho_{i}$, and since gravitational waves may only carry away positive energy $m_{0} \geqslant m=\sum m_{i}$.

Lemma 2.1. Let $a_{i}, b_{i}$ be real numbers, then

$$
\left(\sum a_{i}\right)^{2}+\left(\sum b_{i}\right)^{2} \leqslant\left(\sum \sqrt{a_{i}^{2}+b_{i}^{2}}\right)^{2}
$$

Proof. This follows from the triangle inequality for $n$ points in $\mathbb{R}^{2}$.

Now let $a_{i}=\sqrt{m_{i}^{2}-q_{i}^{2}}$ and $b_{i}=q_{i}$, then we have

$$
\left(\sum \sqrt{m_{i}^{2}-q_{i}^{2}}\right)^{2}+\left(\sum q_{i}\right)^{2} \leqslant\left(\sum m_{i}\right)^{2}
$$

or equivalently

$$
\sum \sqrt{m_{i}^{2}-q_{i}^{2}} \leqslant \sqrt{\left(\sum m_{i}\right)^{2}-\left(\sum q_{i}\right)^{2}}=\sqrt{m^{2}-q^{2}}
$$

Thus we conclude:

$\rho_{0} \leqslant \rho \leqslant \sum \rho_{i} \leqslant \sum m_{i}+\sum \sqrt{m_{i}^{2}-q_{i}^{2}} \leqslant m+\sqrt{m^{2}-q^{2}} \leqslant m_{0}+\sqrt{m_{0}^{2}-q_{0}^{2}}$.

\subsection{The Riemannian Penrose inequality}

The Penrose inequality further simplifies in the time-symmetric case, where the apparent horizon coincides with the outermost minimal surface. Moreover, the dominant energy condition reduces now to non-negative scalar curvature of the Cauchy hypersurface, leading to the Riemannian version of the inequality. 
Theorem 2.2. Let $(M, g)$ be a three-dimensional strongly asymptotically flat Riemannian manifold of non-negative scalar curvature, with an outermost minimal surface boundary $\Sigma$ of area $A=4 \pi \rho^{2}$, then

$$
m \geqslant \frac{1}{2} \rho,
$$

with equality if and only if the manifold is a canonical slice of the Schwarzschild spacetime.

The first published proof of this theorem was given by Huisken-Ilmanen in [12] using inverse mean curvature flow, but required the assumption that $\Sigma$ be connected. Another proof by Bray in [1] used a conformal flow and applied more generally to a non-connected boundary $\Sigma$. Nonetheless, one advantage of the first proof was that it could be immediately generalized to obtain the Einstein-Maxwell case [13], i.e. to prove theorem 1.2 in the case of a connected $\Sigma$. It is important to point out that (2) implies both (1) and a lower bound:

$$
\rho \geqslant m-\sqrt{m^{2}-q^{2}}
$$

In fact this lower bound follows, when $\Sigma$ is connected, from the stability of the outermost horizon [4, 14]. Indeed, the absence of charges outside the horizon, the Cauchy-Schwartz inequality, the charged dominant energy condition, the stability of the outermost horizon, and the Gauss-Bonnet theorem, imply

$$
\begin{aligned}
\frac{4 \pi q^{2}}{\rho^{2}} & =\frac{1}{4 \pi \rho^{2}}\left[\left(\int_{\Sigma} g(E, n) \mathrm{d} A\right)^{2}+\left(\int_{\Sigma} g(B, n) \mathrm{d} A\right)^{2}\right] \\
& \leqslant \int_{\Sigma}\left(|E|^{2}+|B|^{2}\right) \mathrm{d} A \leqslant \int_{\Sigma} \frac{1}{2} R \mathrm{~d} A \leqslant \int_{\Sigma} K=4 \pi N
\end{aligned}
$$

where $R$ is the scalar curvature, and $N, K$, and $n$ are respectively the number of components, the Gauss curvature, and the unit normal of $\Sigma$. In particular one obtains the area-charge inequality $|q| \leqslant \rho$, or more generally

$$
|q| \leqslant \sqrt{N} \rho .
$$

This inequality was obtained in [3]. If $N=1$, the lower bound (6) follows immediately:

$$
m=\sqrt{q^{2}+m^{2}-q^{2}} \leqslant|q|+\sqrt{m^{2}-q^{2}} \leqslant \rho+\sqrt{m^{2}-q^{2}} .
$$

We note that the inequality (8) implies that if $|q|>\sqrt{N} \rho$, then there must be at least $N$ black holes, demonstrating that inequalities between these geometric quantities have topological consequences. All these results are summarized in figure 1.

\subsection{Data for the EAYM equations}

The gauge group for the EAYM matter model is $\otimes_{\ell} U(1)$. The connection, and the field strength $F$, have values in the abelian Lie algebra $\mathbb{R}^{\ell}$. As usual, the electric and magnetic fields are given by $\mathbf{E}=\left(E_{1}, \ldots, E_{\ell}\right)=i_{\mathbf{t}} F, \mathbf{B}=\left(B_{1}, \ldots, B_{\ell}\right)=i_{\mathbf{t}} * F$, where $\mathbf{t}$ is the future directed timelike unit normal to the initial data slice, and $*$ is the Hodge dual. The EAYM equations of motions are 


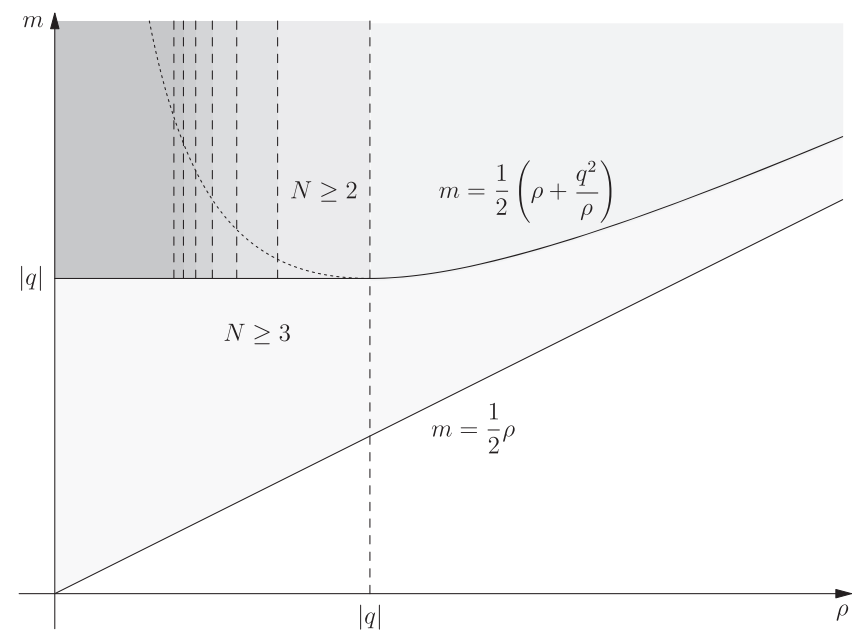

Figure 1. Graphical representation of geometric inequalities.

$$
\begin{aligned}
G_{\mu \nu} & =\bar{R}_{\mu \nu}-\frac{1}{2} \bar{R} \bar{g}_{\mu \nu}=8 \pi T_{\mu \nu}, \\
T_{\mu \nu} & =\frac{1}{4 \pi}\left(F_{\mu \alpha} F_{\nu}^{\alpha}-\frac{1}{4} F_{\alpha \beta} F^{\alpha \beta} \bar{g}_{\mu \nu}\right)=\frac{1}{8 \pi}\left(F_{\mu \alpha} F_{\nu}{ }^{\alpha}+* F_{\mu \alpha} * F_{\nu}{ }^{\alpha}\right), \\
\bar{\nabla}^{\mu} F_{\mu \nu} & =0, \quad \bar{\nabla}^{\mu} * F_{\mu \nu}=0,
\end{aligned}
$$

where barred quantities refer to spacetime objects, and Greek indices run $0, \ldots, 3$. From the expression for the energy-momentum-stress tensor, we obtain the energy density of the slice after contributions from the Yang-Mills fields have been removed:

$$
8 \pi \mu_{\mathrm{YM}}=\bar{R}_{\mathbf{t t}}+\frac{1}{2} \bar{R}-8 \pi T_{\mathbf{t t}}=\frac{1}{2}\left(R-|k|^{2}+(\operatorname{tr} k)^{2}\right)-\left(\sum_{i=1}^{\ell}\left|E_{i}\right|^{2}+\left|B_{i}\right|^{2}\right),
$$

where $k$ is the second fundamental form of the slice. In the time-symmetric case, $k=0$, and the dominant energy condition is

$$
R \geqslant 2\left(\sum_{i=1}^{\ell}\left|E_{i}\right|^{2}+\left|B_{i}\right|^{2}\right)
$$

We note that the dominant energy condition is usually stronger, namely $\mu_{\mathrm{YM}} \geqslant|J|$ where $J_{i}=\frac{1}{8 \pi} G_{\mathbf{t} i}-T_{\mathbf{t} i}$, while here we only use the weaker condition $\mu_{\mathrm{YM}} \geqslant 0$. The total charges are defined by

$$
\begin{aligned}
q_{E_{i}} & =\frac{1}{4 \pi} \int_{S_{\infty}} g\left(E_{i}, n\right) \mathrm{d} A, \quad q_{B_{i}}=\frac{1}{4 \pi} \int_{S_{\infty}} g\left(B_{i}, n\right) \mathrm{d} A, \\
q & =\sqrt{q_{E_{1}}^{2}+q_{B_{1}}^{2}+\cdots+q_{E_{\ell}}^{2}+q_{B_{\ell}}^{2}} .
\end{aligned}
$$




\subsection{Data for the EMD equations}

The EMD action has the Lagrangian

$$
\mathcal{L}=\left(\bar{R}-2 \bar{\nabla}_{\alpha} \phi \bar{\nabla}^{\alpha} \phi-\mathrm{e}^{-2 \phi} F_{\alpha \beta} F^{\alpha \beta}\right) \sqrt{-\operatorname{det} \bar{g}}
$$

There are other EMD models where the coupling $\mathrm{e}^{-2 \phi}$ is replaced by $\mathrm{e}^{-2 a \phi}$, in which $a$ is a coupling constant. While it is possible to conjecture a Riemannian Penrose inequality also for $a \neq 1$, we leave this for future work. From the Lagrangian, one obtains the EMD equations of motion

$$
\begin{aligned}
G_{\mu \nu} & =8 \pi T_{\mu \nu}, \\
T_{\mu \nu} & =\frac{\mathrm{e}^{-2 \phi}}{8 \pi}\left(F_{\mu \alpha} F_{\nu}{ }^{\alpha}+* F_{\mu \alpha} * F_{\nu}{ }^{\alpha}\right)-\frac{1}{4 \pi}\left(\bar{\nabla}_{\mu} \phi \bar{\nabla}_{\nu} \phi-\frac{1}{2} \bar{\nabla}_{\alpha} \phi \bar{\nabla}^{\alpha} \phi \bar{g}_{\mu \nu}\right), \\
\bar{\nabla}^{\mu}\left(\mathrm{e}^{-2 \phi} F_{\mu \nu}\right) & =0, \quad \bar{\nabla}^{\mu} * F_{\mu \nu}=0, \quad \square \bar{g} \phi+\frac{1}{2} \mathrm{e}^{-2 \phi} F_{\mu \nu} F^{\mu \nu}=0 .
\end{aligned}
$$

As above

$$
8 \pi \mu_{\mathrm{MD}}=\frac{1}{2}\left(R-|k|^{2}+(\operatorname{tr} k)^{2}\right)-\mathrm{e}^{-2 \phi}\left(|E|^{2}+|B|^{2}\right)-\left(\partial_{t} \phi\right)^{2}-|\nabla \phi|^{2}
$$

In the time-symmetric case, $k=0$ and $\partial_{t} \phi=0$, thus from $\mu_{\mathrm{MD}} \geqslant 0$ we get the EMD dominant energy condition

$$
R \geqslant 2 \mathrm{e}^{-2 \phi}\left(|E|^{2}+|B|^{2}\right)+2|\nabla \phi|^{2} .
$$

The charges contained in a surface $S$ are given by

$$
\begin{aligned}
& q_{E}(S)=\frac{1}{4 \pi} \int_{S} \mathrm{e}^{-2 \phi} g(E, n) \mathrm{d} A, \quad q_{B}(S)=\frac{1}{4 \pi} \int_{S} g(B, n) \mathrm{d} A \\
& q(S)=\sqrt{q_{E}(S)^{2}+q_{B}(S)^{2}}
\end{aligned}
$$

We note that if $\operatorname{div}\left(\mathrm{e}^{-2 \phi} E\right)=\operatorname{div} B=0$ these charges depend only on the homology class of $S$. The total charges are obtained by taking $S=S_{\infty}$.

A static spherically symmetric EMD black hole was discovered by Gibbons [7, 9]:

$$
\begin{aligned}
\mathrm{d} s^{2} & =-\left(1-\frac{2 m}{r}\right) \mathrm{d} t^{2}+\left(1-\frac{2 m}{r}\right)^{-1} \mathrm{~d} r^{2}+r\left(r-\frac{q^{2}}{m}\right) \mathrm{d} \omega^{2}, \\
F_{r t} & =\frac{q}{r^{2}}, \quad \mathrm{e}^{2 \phi}=1-\frac{q^{2}}{m r},
\end{aligned}
$$

where $\mathrm{d} \omega^{2}$ is the round metric on the unit two-sphere. It was proven to be the unique static solution in $[18,19]$. We note that the scalar curvature of this EMD black hole is given by

$$
R=\frac{1}{r\left(r-q^{2} / m\right)}\left(\frac{2 q^{2}}{r^{2}}+\left(1-\frac{2 m}{r}\right) \frac{q^{4}}{2 m^{2} r\left(r-q^{2} / m\right)}\right),
$$

and hence (10) is an equality in this case. For more details on this solution, see [11]. 


\section{Charges outside the horizon}

Proof of theorem 1.3. The proof follows directly from $[15,16]$, after noting that the only place which requires $E$ and $B$ to be divergence free is when one applies the inverse mean curvature flow at the end of the proof. According to the exhaustion result the flowing surfaces $\Sigma_{t}$ eventually become connected and enclose any large coordinate sphere. Thus, if $E$ and $B$ are divergence free outside of a large coordinate sphere $S_{r}$, then we may apply the inverse mean curvature flow argument once $\Sigma_{t}$ encloses $S_{r}$.

Proof of theorem 1.4. Let the spherically symmetric metric be given by

$$
\mathrm{d} s^{2}=\left(1-\frac{2 m(r)}{r}\right)^{-1} \mathrm{~d} r^{2}+r^{2} \mathrm{~d} \omega^{2}
$$

It is easy to check that $m(r)$ is the Hawking mass of the coordinate sphere $S_{r}$. Furthermore, a horizon occurs at $r=r_{0}$ if $m\left(r_{0}\right)=r_{0} / 2$. If $m(r)$ is increasing there is only one horizon, hence $S_{r_{0}}$ is the outermost horizon. We will use the charged Hawking mass [5]

$$
m_{c}(r)=m(r)+\frac{q^{2}}{2 r}
$$

where $q$ is the total charge. Note that on the horizon, we have

$$
m_{c}\left(r_{0}\right)=\frac{1}{2}\left(r_{0}+\frac{q^{2}}{r_{0}}\right) \text {, }
$$

and at infinity $m_{c}$ tends to the ADM mass $m_{\mathrm{ADM}}$. Clearly, if $m_{c}^{\prime}<0$ for $r>r_{0}$, then (2) will be violated. A violation of (2) with $|q| \leqslant \rho=r_{0}$ implies a violation of (1). Thus, in order to construct a counterexample it suffices to find two functions $m(r)$ and $f(r)$, where $E=f \partial_{r}$, satisfying the following conditions:

(A) The charged dominant energy condition

$$
R=\frac{4 m^{\prime}}{r^{2}} \geqslant 2|E|^{2}=\frac{2 f^{2}}{1-\frac{2 m}{r}} .
$$

(B) Total charge is $q: r^{2} f \rightarrow q$ as $r \rightarrow \infty$.

(C) The condition $|q| \leqslant r_{0}$.

(D) Asymptotic flatness: $m \rightarrow m_{\mathrm{ADM}}$ as $r \rightarrow \infty$.

(E) The inequality

$$
m_{c}^{\prime}=m^{\prime}-\frac{q^{2}}{2 r^{2}}<0 .
$$

Choose $0<q<r_{0}$. We begin by solving

$$
m^{\prime}=\frac{q^{2}}{2 r^{2}}\left(1-\frac{r_{0}}{r}\right), \quad m\left(r_{0}\right)=\frac{r_{0}}{2},
$$


so that $m^{\prime}>0$ and (14) is satisfied for $r>r_{0}$. One finds

$$
m=\frac{r_{0}}{2}+\frac{q^{2}}{4 r_{0}}-\frac{q^{2}}{2 r}+\frac{q^{2} r_{0}}{4 r^{2}}
$$

Clearly asymptotic flatness is satisfied, and in fact

$$
m_{\mathrm{ADM}}=\frac{r_{0}}{2}+\frac{q^{2}}{4 r_{0}}
$$

Furthermore $2 m^{\prime}-1<0$, and consequently $2 m<r$, for $r>r_{0}$. Finally observe that

$$
h(r):=\frac{2 m^{\prime}}{r^{2}}\left(1-\frac{2 m}{r}\right)=\frac{q^{2}}{r^{4}}\left(1-\frac{r_{0}}{r}\right)\left(1-\frac{2 m}{r}\right) .
$$

It follows that we can find a function $f(r)$ satisfying $f\left(r_{0}\right)=0$, the charged dominant energy condition $f(r)^{2}<h(r)$ for $r>r_{0}$, and $r^{2} f(r) \rightarrow q$ as $r \rightarrow \infty$.

Proof of proposition 1.5. Let $\left(M, g_{0}, E_{0}\right)$ denote the canonical slice of the ReissnerNordström spacetime, and let $r$ denote the anisotropic radial coordinate for this data. Consider the annulus $\Omega\left(r_{1}, r_{2}\right)$ where $r_{2}>r_{1}>m+\sqrt{m^{2}-q^{2}}$, and fix $f \in C_{c}^{\infty}\left(\Omega\left(r_{1}, r_{2}\right)\right)$ to be nonnegative and not identically zero. Since the scalar curvature $R_{g_{0}}$ is nonconstant in $\Omega\left(r_{1}, r_{2}\right)$, the formal $L^{2}$-adjoint of the linearized scalar curvature operator has trivial kernel. It then follows from [2] that there exists a smooth contravariant 2-tensor $h \in C_{c}^{\infty}\left(\Omega\left(r_{1}, r_{2}\right)\right)$, such that the scalar curvature of the metric $\tilde{g}=g_{0}+\varepsilon h$ is given by $R_{\tilde{g}}=R_{g_{0}}+\varepsilon f$ for $\varepsilon>0$ sufficiently small. It is also clear from the proof in [2] that if $f$ is chosen to be spherically symmetric, then $h$ is also spherically symmetric.

Define $\tilde{E}=E_{0}+\varepsilon V$, and observe that

$$
|\tilde{E}|_{\tilde{g}}^{2}=\left|E_{0}\right|_{g_{0}}^{2}+2 \varepsilon g_{0}\left(E_{0}, V\right)+\varepsilon h\left(E_{0}, E_{0}\right)+O\left(\varepsilon^{2}\right) .
$$

Since $E_{0}=-q \nabla r^{-1}$, the implicit function theorem may be used to find $V$ such that $|\tilde{E}|_{\tilde{g}}^{2}=\left|E_{0}\right|_{g_{0}}^{2}$. Therefore

$$
R_{\tilde{g}}=R_{g_{0}}+\varepsilon f=2\left|E_{0}\right|_{g_{0}}^{2}+\varepsilon f=2|\tilde{E}|_{\tilde{g}}^{2}+\varepsilon f \geqslant 2|\tilde{E}|_{\tilde{g}}^{2},
$$

so that the initial data set $(M, \tilde{g}, \tilde{E})$ satisfies the charged dominant energy condition. Moreover, it is clear that this initial data set satisfies all desired hypotheses, except possibly the outermost condition for the minimal surface boundary. However, it can easily be seen that the minimal boundary is outermost by choosing $\varepsilon$ sufficiently small. Namely, by choosing $\varepsilon$ sufficiently small, the mean curvature of each coordinate sphere $S_{r}, r>m+\sqrt{m^{2}-q^{2}}$, remains positive. Thus, the exterior region is foliated by surfaces of positive mean curvature, showing that no other minimal surface enclosing the boundary can exist (by the maximum principle for minimal surfaces).

\section{Other matter models}

Proof of theorem 1.6. Apply a rotation

$$
\left(E_{1}, B_{1}, \ldots, E_{\ell}, B_{\ell}\right) \mapsto\left(\tilde{E}_{1}, \tilde{B}_{1}, \ldots, \tilde{E}_{\ell}, \tilde{B}_{\ell}\right),
$$


so that all the transformed charges $q_{\tilde{E}_{i}}$ and $q_{\tilde{B}_{i}}$ vanish except possibly $q_{\tilde{E}_{1}}$, and $q_{\tilde{E}_{1}}=q$. If $q \geqslant \rho$ then the positive mass theorem with charge applies, and we get (1) as explained in the introduction. Otherwise, starting with this new data, one may apply the proof in [15], conformally deforming all the electromagnetic fields in the same manner $\tilde{E}_{i}(t)=u_{t}^{-6} \tilde{E}_{i}$, $\tilde{B}_{i}(t)=u_{t}^{-6} \tilde{B}_{i}$. Both the charged conformal flow and the inverse mean curvature flow arguments now proceed as in theorem 1.3, and we obtain (2). If there are no charges and equality holds, we obtain as in [15] that the transformed data is a canonical slice of ReissnerNordström. After rotating back, we find that the original data is a canonical slice of an EAYM Reissner-Nordström black hole.

Discussion on conjecture 1.7. An initial data set for the EMD equations consists of $(M, g, k, E, B, \phi, \psi)$, where $\psi$ is the initial time derivative of $\phi$. In the time-symmetric case $k$ and $\psi$ vanish. We assume the data is strongly asymptotically flat, satisfies the EMD dominant energy condition (10), and that charges are absent, $\operatorname{div}\left(\mathrm{e}^{-2 \phi} E\right)=\operatorname{div} B=0$ outside the horizon, or more generally outside a compact set.

Our first observation is that a Penrose heuristic argument, as in section 2.2, is still valid. Start from data with paramaters $m_{0}, \rho_{0}, q_{0}$, and assume the data settles to an EMD black hole as in (11) with parameters $m, \rho$, and $q$. Then, the charge is conserved $q=q_{0}$, the Hawking area theorem applies giving $\rho_{0} \leqslant \rho$, and as before the masses satisfy $m \leqslant m_{0}$. Since for the EMD black hole we have

$$
m=\frac{1}{2} \sqrt{\rho^{2}+2 q^{2}}
$$

it follows that

$$
m_{0} \geqslant m=\frac{1}{2} \sqrt{\rho^{2}+2 q^{2}} \geqslant \frac{1}{2} \sqrt{\rho_{0}^{2}+2 q_{0}^{2}},
$$

and hence (4) holds for the initial data.

We now point out a number of significant differences between this setting and our work in [15]. First, there are analogs of the Majumdar-Papapetrou multiple neck solutions [6], but they do not pose an obstruction to the lower bound (4). Indeed, the right hand side of (4) is always monotone in $\rho$ unlike the right hand side of (2), hence we do not expect an auxiliary inequality, such as the area/charge inequality in theorem 1.2, to be necessary to prove (4). These extremal solutions have, in a conformally related 'string' metric, asymptotically cylindrical infinitely long necks just as in Majumdar-Papapetrou, but in the spacetime metric these have cross sectional area tending to zero at a finite distance. Since we have $\rho=0$ in this case, with $q \neq 0$, there are EMD black holes with nearby parameters that have a single connected component horizon, and charge to area radius ratio arbitrarily small. Thus the phenomenon described in section 2.3 whereby excess charge leads to high multiplicity of horizon components is absent in the EMD model. The mathematical reason is that in the EMD case, to replace the integral over $S$ with the integral over $S_{\infty}$ in inequality (7), would require $\mathrm{e}^{-\phi} E$ and $\mathrm{e}^{-\phi} B$ to be divergence free rather than, as is assumed, $\mathrm{e}^{-2 \phi} E$ and $B$.

Notwithstanding these observations, the many similarities we now outline, lead us to surmise that the same approach used in [15] should apply in the EMD setting. First, a positive mass theorem with charge holds [20, 23].

Theorem 4.1. Let $(M, g, k, E, B, \phi, \psi)$ be a strongly asymptotically flat initial data set for the EMD equations with an apparent horizon boundary, satisfying the EMD dominant energy condition, with ADM mass $m$ and total charge $q$. If charge densities $\operatorname{div}\left(\mathrm{e}^{-2 \phi} E\right)$ and $\operatorname{div} B$ 
vanish, then

$$
m \geqslant \frac{1}{\sqrt{2}}|q|
$$

Observe that the initial data is not assumed to be time-symmetric. On the other hand, the rigidity statement has not yet been proved. We suspect that in analogy with the EM case, equality holds if and only if the data can be embedded as a Cauchy slice in one of the GHS static extremal EMD solutions from [6], and if the data is time-symmetric equality holds if and only if that slice is the cannonical slice. That said, contrary to [15], theorem 4.1 likely plays no role in the proof of (4). Instead, we believe that an EMD charged Hawking mass $m_{\mathrm{D}}$ exists as in [5] satisfying the following properties: (i) $m_{\mathrm{D}}$ is monotonically non-decreasing under the Huisken-Ilmanen weak inverse mean curvature flow, and it is constant only for the central spheres in the spherically symmetric EMD black hole; (ii) $m_{\mathrm{D}}$ converges to $\frac{1}{2} \sqrt{\rho^{2}+2 q^{2}}$ on the outermost horizon, and to the ADM mass at infinity. This would prove conjecture 1.7 including the rigidity statement, provided the horizon is connected and there are no charges outside the horizon. Finally, we conjecture that there exists an EMD conformal flow satisfying: (i) the EMD dominant energy condition is preserved under the flow; (ii) the absence of charges is preserved under the flow; (iii) the ADM mass is monotonically nonincreasing under the flow and constant only for the spherically symmetric EMD black holes; (iv) the area of the boundary is constant under the flow; (v) the boundary eventually encloses any compact set on the original manifold. This would prove the full conjecture. For (iii), a critical ingredient, used in both [1] and [15], namely a conformal factor used to prove uniqueness in a doubling argument, is already available [18, 19].

\section{Acknowledgments}

MK acknowledges the support of NSF grants DMS-1007156 and DMS-1308753. SY acknowledges the support of JSPS grants 23654061 and 24340009.

\section{References}

[1] Bray H L 2001 Proof of the Riemannian Penrose inequality using the positive mass theorem J. Differ. Geom. 59 177-267

[2] Corvino J 2000 Scalar curvature deformation and a gluing construction for the Einstein constraint equations Commun. Math. Phys. 214 137-89

[3] Dain S, Jaramillo J L and Reiris M 2012 Area-charge inequality for black holes Class. Quantum Grav. 29035013

[4] Dain S, Khuri M A, Weinstein G and Yamada S 2013 Lower bounds for the area of black holes in terms of mass charge and angular momentum Phys. Rev. D 88024048

[5] Disconzi M M and Khuri M A 2012 On the Penrose inequality for charged black holes Class. Quantum Grav. 29245019

[6] Garfinkle D, Horowitz G T and Strominger A 1991 Charged black holes in string theory Phys. Rev. D 43 3140-3

[7] Gibbons G W 1982 Antigravitating black hole solitons with scalar hair in $N=4$ supergravity Nucl. Phys. B 207 337-49

[8] Gibbons G W, Hawking S W, Horowitz G T and Perry M J 1983 Positive mass theorems for black holes Commun. Math. Phys. 88 295-308

[9] Gibbons G W and Maeda K 1988 Black holes and membranes in higher dimensional theories with dilaton fields Nucl. Phys. B 298741 
[10] Hawking S W and Ellis G F R 1973 Cambridge monographs on mathematical physics The Large Scale Structure of Space-Time (London: Cambridge University Press)

[11] Horowitz G T 1992 The dark side of string theory: black holes and black strings Proc. 1992 Trieste Spring School on String Theory and Quantum Gravity

[12] Huisken G and Ilmanen T 2001 The inverse mean curvature flow and the Riemannian Penrose inequality J. Differ. Geom. $59353-437$

[13] Jang P S 1979 Note on cosmic censorship Phys. Rev. D 20 834-8

[14] Khuri M A, Weinstein G and Yamada S 2012 On the Riemannian Penrose inequality with charge and the cosmic censorship conjecture Res. Inst. Math. Sci. Kokyuroku $186263-66$

[15] Khuri M A, Weinstein G and Yamada S 2014 Proof of the Riemannian Penrose inequality with charge for multiple black holes arXiv:1409.3271

[16] Khuri M A, Weinstein G and Yamada S 2014 The Riemannian Penrose inequality with charge for multiple black holes Proc. Complex Analysis and Dynamical System 6th

[17] Malec E and Murchadha N Ó 1994 Trapped surfaces and the Penrose inequality in spherically symmetric geometries Phys. Rev. D 49 6931-4

[18] Mars M and Simon W 2002 On uniqueness of static Einstein-Maxwell-dilaton black holes Adv. Theor. Math. Phys. 6 279-305

[19] Masood-ul Alam A K M 1993 Uniqueness of a static charged dilaton black hole Class. Quantum Grav. 102649

[20] Nozawa M 2011 On the Bogomol'nyi bound in Einstein-Maxwell-dilaton gravity Class. Quantum Grav. 28175013

[21] Penrose R 1973 Naked Singularities Ann. New York Acad. Sci. 224 125-34

[22] Penrose R 1982 Some unsolved problems in classical general relativity Seminar on Differential Geometry Ann. Math. Stud. 102 (Princeton, NJ: Princeton University Press) pp 631-68

[23] Rogatko M 2000 Positive mass theorem for black holes in Einstein-Maxwell axion-dilaton gravity Class. Quantum Grav. 17 11-17

[24] Weinstein G and Yamada S 2005 On a Penrose inequality with charge Commun. Math. Phys. 257 703-23 